\title{
BMJ Global Health Comparison of community-led distribution of HIV self-tests kits with distribution by paid distributors: a cluster randomised trial in rural Zimbabwean communities
}

Euphemia Lindelwe Sibanda (D) , ${ }^{1,2}$ Collin Mangenah (D) , ${ }^{1}$ Melissa Neuman (D) , ${ }^{3}$ Mary Tumushime, ${ }^{1}$ Constancia Watadzaushe, ${ }^{1}$ Miriam N Mutseta, ${ }^{4}$ Galven Maringwa, ${ }^{1}$ Jeffrey Dirawo, ${ }^{1}$ Katherine L Fielding (D) , 3,5 Cheryl Johnson (i) , ${ }^{6}$ Getrude Ncube, ${ }^{7}$ Miriam Taegtmeyer (i) , ${ }^{2}$ Karin Hatzold, ${ }^{8}$ Elizabeth Lucy Corbett (D) , 9,10 Fern Terris-Prestholt (D) ,11,12 Frances M Cowan (1) ${ }^{1,2}$

To cite: Sibanda EL, Mangenah C, Neuman M, et al. Comparison of community-led distribution of HIV self-tests kits with distribution by paid distributors: a cluster randomised trial in rural Zimbabwean communities. BMJ Global Health 2021;6:e05000. doi:10.1136/ bmjgh-2021-005000

Handling editor Seye Abimbola - Additional supplemental material is published online only. To view, please visit the journal online (http://dx.doi.org/10. 1136/bmjgh-2021-005000).

Received 12 January 2021 Accepted 18 June 2021
Check for updates

(C) World Health Organization 2023. Licensee BMJ.

For numbered affiliations see end of article.

Correspondence to

Dr Euphemia Lindelwe Sibanda euphemia@ceshhar.co.zw

\section{ABSTRACT}

Background We compared community-led versus an established community-based HIV self-testing (HIVST) model in rural Zimbabwe using a cluster-randomised trial. Methods Forty village groups were randomised 1:1 using restricted randomisation to community-led HIVST, where communities planned and implemented HIVST distribution for 4 weeks, or paid distribution (PD), where distributors were paid US\$50 to distribute kits door-todoor over 4 weeks. Individual level primary outcomes compared household survey responses by arm 4 months post-intervention for: (1) newly diagnosed HIV during/ within 4 months following HIVST distribution, (2) linkage to confirmatory testing, pre-exposure prophylaxis or voluntary medical male circumcision during/within 4 months following HIVST distribution. Participants were not masked to allocation; analysis used masked data. Trial analysis used random-effects logistic regression.

Distribution costs compared: (1) community-led HIVST, (2) PD HIVST and (3) PD costs when first implemented in 2016/2017.

Results From October 2018 to August 2019, 27812 and 36699 HIVST kits were distributed in community-led and PD communities, respectively. We surveyed 11150 participants and 5683 were in community-led arm. New HIV diagnosis was reported by 211 (3.7\%) community-led versus 197 (3.6\%) PD arm participants, adjusted OR (aOR) 1.1 (95\% Cl 0.72 to 1.56$) ; 318$ (25.9\%) community-led arm participants linked to post-test services versus 361 (23.9\%) in PD arm, aOR 1.1 (95\% Cl 0.75 to 1.49. Cost per HIVST kit distributed was US\$6.29 and US\$10.25 for PD and community-led HIVST, both lower than 2016/2017 costs for newly implemented PD (US\$14.52). No social harms were reported.

Conclusions Community-led HIVST can perform as well as paid distribution, with lower costs in the first year. These costs may reduce with programme maturity/learning. Trial registration number PACTR201811849455568.

\section{WHAT IS ALREADY KNOWN?}

$\Rightarrow$ Community-based HIV self-testing (HIVST) is feasible and acceptable and improves uptake of testing by groups who would not otherwise test.

$\Rightarrow$ However, it is resource intensive and there is need to find lower cost models for community-based HIVST distribution.

$\Rightarrow$ Community-led models have been successfully used to drive various health interventions.

$\Rightarrow$ They have potential to successfully implement community-based HIVST at lower cost.

$\Rightarrow$ We compared community-led HIVST with an established community-based HIVST distribution model in rural Zimbabwe using a cluster-randomised trial.

\section{WHAT ARE THE NEW FINDINGS?}

$\Rightarrow$ Communities were able to develop and implement their own HIVST models to produce outcomes that were comparable to those of a programme where HIVST kits were distributed by distributors who received stipends and professional support.

$\Rightarrow$ The cost of distributing HIVST kits in the mature paid distributor model were lower than those of the community-led model.

$\Rightarrow$ Of note, however, community-led distribution costs were lower than those of the paid distribution model when it was first introduced in 2016-2017, indicating that as the programme matures the costs may come down.

\section{WHAT DO THE NEW FINDINGS IMPLY?}

$\Rightarrow$ The community-led HIVST intervention is a promising model that can be integrated with local standard of care and for scale up of self-testing.

$\Rightarrow$ Additional research can explore layering of different interventions that have potential to be driven by communities. 


\section{INTRODUCTION}

HIV self-testing (HIVST) is recommended as a testing strategy by WHO. ${ }^{1}$ Various models of distributing HIVST kits have been implemented and/or evaluated, including community-based, health facility-based, pharmacy, workplace, secondary distribution and distribution through programmes such as voluntary medical male circumcision (VMMC) and antenatal care. ${ }^{2-5}$ As has been found for provider-delivered community-based HIV testing services, ${ }^{6} 7$ there is evidence that community-based HIVST is acceptable ${ }^{89}$ and can increase uptake of testing and linkage to HIV treatment, including among groups who typically have lower uptake of testing, such as men and young people. ${ }^{4510}$ However, provision of communitybased HIVST can be resource-intensive, particularly when kits are delivered door-to-door. ${ }^{11}$ As programmes prepare for full national scale up, there is need to identify and evaluate sustainable and/low cost models of providing HIVST in communities. A potential model is the community-led approach.

Community-led interventions are designed and implemented by communities, usually with support from programme implementers. ${ }^{12}$ They have been used to drive various health interventions, ${ }^{12-15}$ with success depending on the extent to which communities take ownership of the intervention and are motivated by perceived community benefits to take collective action towards ensuring implementation. Previous research on HIVST distribution in rural areas found that briefly trained door-to-door paid HIVST community-based distributors were able to reach $50.3 \%$ of adults with detectable impact on demand for antiretroviral therapy (ART) ${ }^{10}$ A small incentive paid to increase post-test linkage did not motivate distributors beyond that achieved by a fixed stipend, with distributors stating the importance of non-monetary drivers of job satisfaction such as standing in the community and importance of the task, previously associated with successful unpaid community-led interventions. A community-led HIVST approach might be able to improve engagement of communities with awareness of HIV prevention messages, notably ' $\mathrm{U}=\mathrm{U}$ ' messaging around treatment-asprevention that is particularly relevant for HIVST given the adverse event profile for discordant couples, and also pre-exposure prophylaxis (PrEP) and VMMC. The community-led approach may also target the distribution of kits towards people who need to test. Finally, community-led HIVST has potential to reduce resource requirements from the provider's perspective.

We used a cluster-randomised trial design (because the intervention could only be implemented at community level) to directly compare outcomes and costs of community-led HIVST with a more vertical approach of professionally-supervised paid community-based distributors, with the following primary objectives pertaining to the individual level: to determine the effect of community-led HIVST on (1) recent HIV diagnoses, (2) linkage to post-test HIV care and prevention services and (3) to compare the distribution costs of community-led models against those of newly introduced and repeat rounds of paid distributor HIVST campaigns. ${ }^{11}$

\section{METHODS}

This research is part of the large portfolio of studies conducted by the Self Testing AfRica (STAR) Initiative, http://hivstarlshtmacuk/. ${ }^{16}$ Implementation of HIVST distribution was led by Population Services International (PSI) between October 2018 and August 2019, on a rolling campaign basis as we moved from one community to the next.

\section{Population and setting}

The study was conducted in Zimbabwe, a country with HIV prevalence of $12.9 \% .{ }^{17}$ Zimbabwe Ministry of Health and Child Care provides decentralised HIV services across all levels of care, with facility-based and community-based support given by implementing partners across many districts. Study implementation was done in six rural districts (Shamva, Muzarabani, Mutoko, Zvimba, Shurugwi and Umguza) in five provinces where PSI was implementing HIV testing services. Clusters were defined as headman units, an official administrative subunit through which rural community-level activities are implemented in Zimbabwe. Because headman unit sizes varied, potentially eligible headmen units had to have at least three census enumeration areas (CEAs) within. We randomly selected one headman unit of at least 3 CEAs per ward, (figure 1). The minimum distance between each headman unit and its nearest neighbour was $20 \mathrm{~km}$.

\section{Study design}

We conducted a cluster randomised trial comparing two HIVST distribution strategies, as summarised in figure 1. Headman units were randomised to either community-led or paid HIVST distribution. Distribution was done over 4 weeks, with flexible distribution days and times according to distributor preference and social etiquette. In general distributors undertook distribution on several days of the week.

Trial outcomes were evaluated using household surveys 4 months post-distribution to determine uptake of HIVST and linkage to post-test services.

Monthly clinic-level data of new ART initiations for all facilities across the six districts were also used to evaluate the impact of HIVST using a difference-in-differences design (6 months before HIVST distribution began, during and 3 months after). We conducted detailed costing of the community-led model and compared it with costs of the (1) the paid distributor model that was implemented during the study and, (2) a historical paid distributor model when first implemented in 2016/17.

\section{Detailed procedures}

\section{Randomisation and masking}

The unit of randomisation was the headman unit. Randomisation of headman units (1:1) by the study statistician 


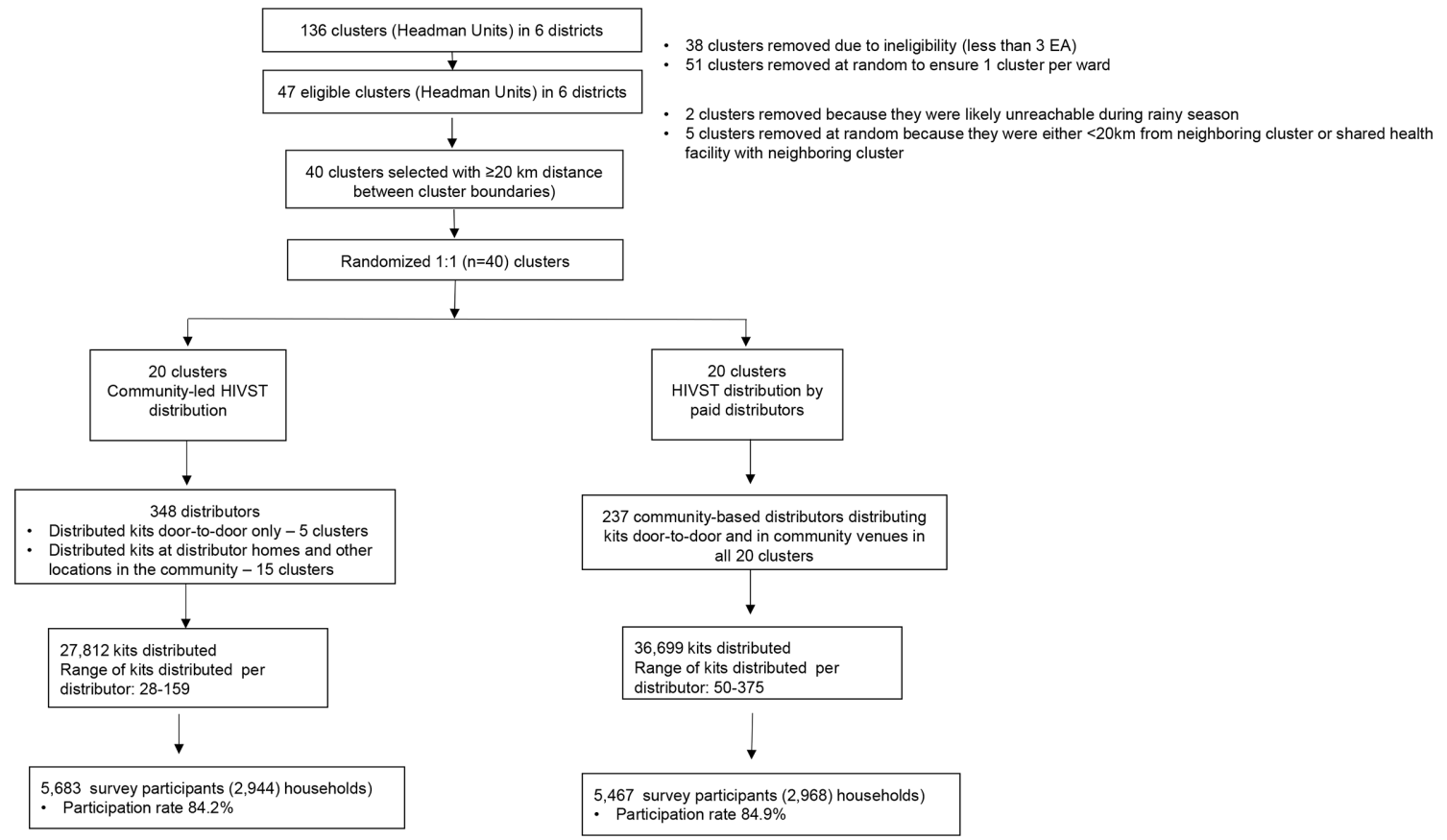

Figure 1 Trial profile. EA, enumeration area; HIVST, HIV self-testing.

(MN) was restricted to ensure balance across arms by district, distance from the centre of the headman unit to the nearest health facility and availability of PrEP at the nearest health facility. Out of all the possible combinations, 100000 randomisation options were generated at random and were subjected to the restriction criteria, resulting in 18013 options, from which the final randomisation option was randomly selected by the statistician and communicated to the implementing team. No allocation concealment was done.

Headman units and distributors could not be masked to study arm, but data and investigators were masked until analysis of primary outcomes was complete.
Community-led HIVST distribution (community-led arm)

The intervention was implemented at the headman unit level. Figure 2 illustrates the process followed to engage headman units, piloted in four headman units before the trial began. A detailed description of the intervention according to the Template for Intervention Description and Replication (TIDieR) framework $^{18}$ is given in figure 3. First, PSI met with community leadership to explain the concept of a community-led approach, including the potential benefits and challenges. It was explained that headman units would need to invest their own resources, particularly time, with no financial

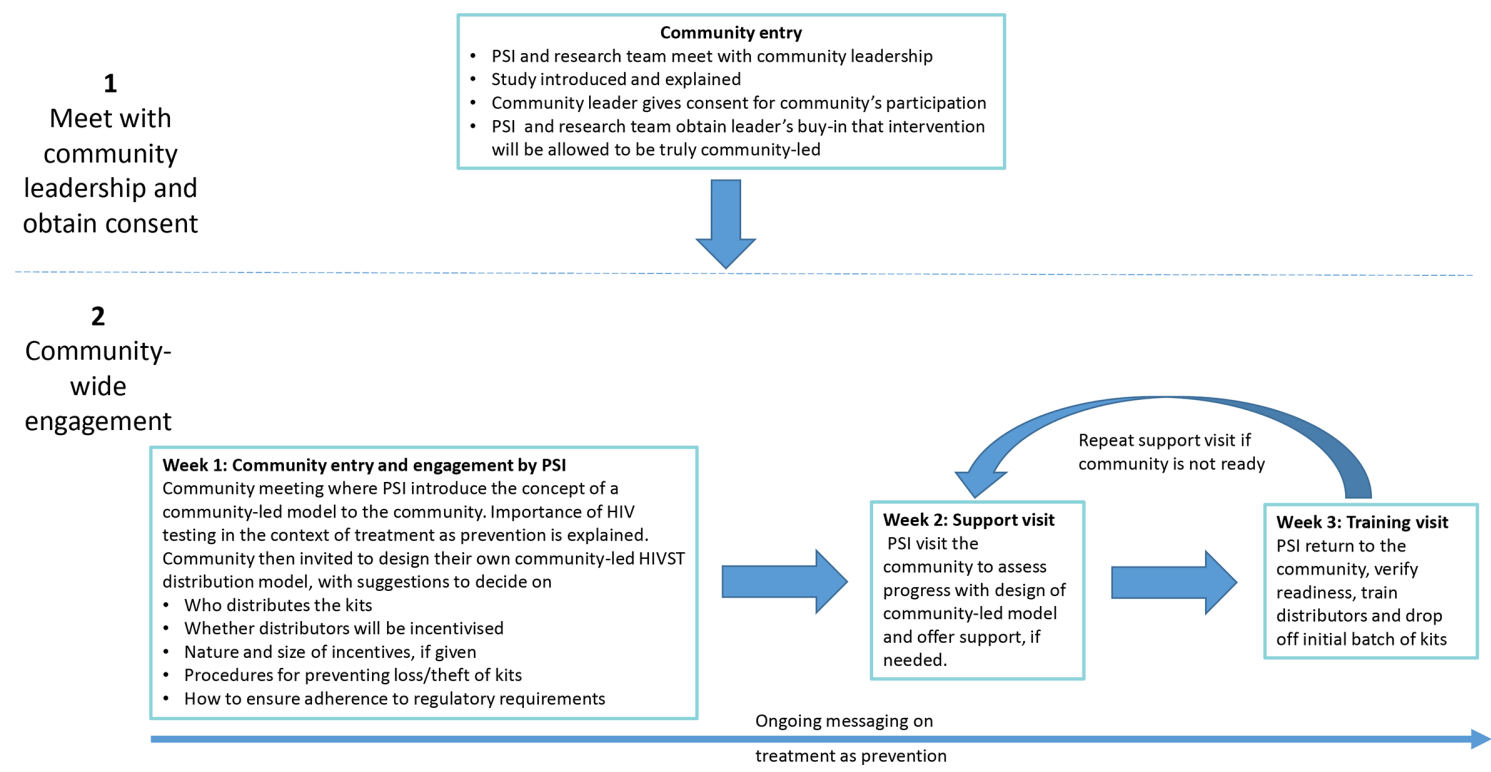

Figure 2 Process of engaging community-led HIVST clusters. HIVST, HIV self-testing; PSI, Population Services International. 


\begin{tabular}{|c|c|c|}
\hline ITEM & INTERVENTION ARM & COMPARISON ARM \\
\hline 1. Brief name & Community-led distribution of HIV self-test kits (HIVST) & Community-based distribution of HIVST by paid distributors \\
\hline 2. Why & $\begin{array}{l}\text { Community-based HIVST can increase uptake of testing (including among first time testers) and } \\
\text { linkage to post-test services. Distributing HIVST using paid distributors (comparison arm) can be } \\
\text { expensive and therefore more challenging to scale-up. Also, in a community-led approach, } \\
\text { communities can target testing and messaging on HIV treatment to people who needed these } \\
\text { services most. }\end{array}$ & \\
\hline \multicolumn{3}{|l|}{ What } \\
\hline 3. Materials & $\begin{array}{l}\text { For distributors: Training mate rials providing information on HIV and HIVST, the effectiveness of HIV } \\
\text { treatment for preventing transmission, supporting others to use HIVST kits, providing information to } \\
\text { promote and support linkage to appropriate post-test services (ART, PrEP, VMMC), use of paper- } \\
\text { based data capture tools. } \\
\text { For community: Posters and flyers promoting HIVST and giving information about the effectiveness } \\
\text { of HIV treatment for preventing transmission. } \\
\text { These materials are available upon request from PSI Zimbabwe } \\
\text { - no incentive for community or individual distributers }\end{array}$ & $\begin{array}{l}\text { For distributors: Training materials providing information on HIV and HIVST, supporting } \\
\text { others to use HIVST kits, providing information to promote and support linkage to } \\
\text { appropriate post-test services, use of electronic (tablets) data capture tools } \\
\text { These materials are available upon request from PSI Zimbabwe } \\
\text { Fixed stipend of } \$ 50 \text { at the end of the distribution period }\end{array}$ \\
\hline 4. Procedures & \multicolumn{2}{|c|}{ See description in text } \\
\hline $\begin{array}{l}\text { 5. WHO } \\
\text { provided }\end{array}$ & $\begin{array}{l}\text { PSI staff - Staff qualified in social sciences and counselling with experience of supporting } \\
\text { implementation of HIV services in communities. } \\
\text { Distributors - Residents of communities where distribution happened. They were selected by the } \\
\text { community because they had good community standing and were literate. Individuals of different } \\
\text { characteristics filled this role. }\end{array}$ & $\begin{array}{l}\text { PSI staff - Staff qualified in social sciences and counselling with experience of } \\
\text { supporting implementation of HIV services in communities } \\
\text { Distributors - Residents of communities where distribution happened. They were } \\
\text { selected by community leaders because they had previous experience of working with } \\
\text { HIV programs, community work, good literacy levels and good standing in the } \\
\text { community. }\end{array}$ \\
\hline 6. How & \multicolumn{2}{|l|}{$\begin{array}{l}\text { Communities were engaged by PSI in face-to-face group meetings } \\
\text { Distributors provided HIVST individually }\end{array}$} \\
\hline 7. Where & \multicolumn{2}{|c|}{$\begin{array}{l}\text { The intervention happened in } 20 \text { clusters per arm. A cluster was defined as a headman unit - a group of neighbouring villages } \\
\text { across the country. }\end{array}$} \\
\hline $\begin{array}{l}\text { 8. When/ how } \\
\text { much }\end{array}$ & \multicolumn{2}{|c|}{ Distribution was done between October 2018 and August 2019. Each cluster only distributed kits over one campaign period. } \\
\hline 9. Tailoring & $\begin{array}{l}\text { Each community was asked to design a model of HIVST distribution that suited them to maximise } \\
\text { acceptability, ease of implementation and uptake of testing. Distributors could distribute kits door- } \\
\text { to-door, people could collect kits from distributors' homes or at different community locations, or } \\
\text { communities could implement models that combined all or any of the above. }\end{array}$ & None \\
\hline $\begin{array}{ll}10 . \\
\text { Modifications }\end{array}$ & \multicolumn{2}{|l|}{ No changes } \\
\hline \multicolumn{3}{|l|}{ How well } \\
\hline $\begin{array}{l}\text { 11. How well } \\
\text { planned }\end{array}$ & $\begin{array}{l}\text { The local clinic provided support and supervision of distributors to ensure correct implementation. } \\
\text { They provided HIV kit replenishments - they reviewed distribution records and only supplied } \\
\text { additional stock if records were satisfactory. } \\
\text {-Social science researchers conducted participant observations to explore fidelity of implementation. }\end{array}$ & $\begin{array}{l}\text { PSI provided support and supe rvision of distributors to ensure correct implementation. } \\
\text { This included phone calls, support visits and unannounced spot checks of HIVST stocks. } \\
\text { PSI also provided kit replenishment. } \\
\text {-Social science researchers conducted participant observations to explore fidelity of } \\
\text { implementation. }\end{array}$ \\
\hline 12. Actual & $\begin{array}{l}\text { Adherence to arm assignment was reported in all } 20 \text { headman units. Implementation was done with } \\
\text { integrity as reported by clinics. Quality of program delivery differed across headman units: some } \\
\text { distributors took long to replenish their stocks, and in some headman units there was no evidence of } \\
\text { widespread distribution of posters and flyers. }\end{array}$ & $\begin{array}{l}\text { Adherence to arm assignment was reported in all } 20 \text { headman units. Im plementation } \\
\text { was done with integrity as reported by PSI. Quality of program delivery was } \\
\text { satisfactory as reported by PSI. }\end{array}$ \\
\hline
\end{tabular}

Figure 3 Template for Intervention Description and Replication (TIDieR) table for describing the community-led and paid distribution HIV self-test models. ART, antiretroviral therapy; PrEP, pre-exposure prophylaxis; PSI, Population Services International; VMMC, voluntary medical male circumcision.

compensation and that headman units and their leadership could decline participation. Because communitylevel interventions typically follow a top-down approach, we obtained the leadership's buy-in that they would not impose their ideas, but would support a truly community-led intervention. Leadership was then asked to set up community-level meetings to facilitate engagement of PSI with the headman unit.

All headman unit members aged 16 years and above were invited to the first meeting (16 years being the minimum age for consenting to an HIV test in Zimbabwe) during which we introduced the concept of a community-led model and described the importance of HIV testing and the concept of ART for preventing HIV transmission. ${ }^{19}$ We explained that new HIV infections in their headman unit could be reduced by early HIV testing and linkage to ART. Other HIV prevention interventions (VMMC and PrEP) were also discussed.

Headman units were then tasked with designing their own models of distributing HIVST kits, ensuring that their models adhered to the following Ministry of Health guidelines: (1) no kits given to individuals younger than 16 years, (2) no forced testing or results disclosure, (3) confidentially was to be upheld. Headman units were given 1 week for internal consultations, after which PSI visited the named contacts to describe the agreed model, and verify that it met the Ministry of Health guidelines. If the model and agreed distributors were deemed ready, training for HIVST distributors was scheduled for the following week, otherwise headman units were revisited 1 week later.

HIVST distributors were trained over 3 days, according to Ministry of Health guidelines, and given an initial batch of 50 HIVST kits for distribution with additional kits available from the local clinic. Training covered information on HIV testing, supporting others to use HIVST kits, providing information to promote and support linkage to appropriate post-test services, providing information on effectiveness of antiretroviral therapy for HIV prevention, and use of data capture tools. During training role plays were also done to enhance distributor readiness for the task. Distributors were given 4-6 weeks to complete distribution. Headman units were asked to ensure that kits were given to all willing individuals (one kit per person) who met the testing criteria above. Distributors told kit recipients about post-test services they could access from local clinics: confirmatory testing for those who got reactive results and PrEP or VMMC for those who considered themselves at risk of HIV and had HIV negative self-test results (this information was also written on the instructions for use). Recipients of test kits could self-test at their convenience and did not need to disclose their results 
to distributors. All HIVST kits came with instructions for use in English and the two major local languages in Zimbabwe. In addition, distributors conducted in-person demonstration of testing procedures, including explanation of how to read and interpret the results. Our earlier work to refine self-test instructions for the Zimbabwean context helped ensure accurate testing. ${ }^{20}$ During distribution, ongoing support and supervision of distributors was provided by the local clinic. This included answering any questions and checking that distribution records were complete. Local clinics also provided kit replenishments.

\section{Distribution by paid distributors}

A detailed description of HIVST distribution by paid distributors according to the TIDieR framework ${ }^{18}$ is given in figure 3 . This used a previously described model ${ }^{10}$ (that was run in communities that were different from the communities in this trial) where community leadership identified individuals who could distribute HIVST kits in their communities. Distributors received the same 3-day training as described above, and had 4 weeks to cover a defined geographical area (one or two villages) using door-to-door distribution, with expectation that they would give kits to all willing individuals (one kit per person) in their geographical area who met the testing criteria. They also talked about linkage to post-test services as described above for distributors in the community-led model. PSI trained and supported distributors with kits and a one-off stipend of US\$50.

\section{Methods for detecting social harms during HIVST distribution}

During HIVST distribution in both arms, surveillance for social harms was done using four methods: (1) assessment of reports shared by organisations providing support for victims of gender-based violence in the study communities, (2) reports to the toll-free number that was manned by PSI, (3) self-reports in the survey and, (4) reports by distributors.

\section{Intervention evaluation}

Four months after HIVST distribution, populationrepresentative household surveys were conducted in all study areas. All eligible individuals (aged $\geq 16$ years and resided in the study community during HIVST distribution in households within three enumeration areas (EAs) per headman unit) were asked to provide written consent for self-administered Audio Computer-Assisted Survey Instrument (ACASI) questionnaire, with random selection used for headman units with $\geq 3$ EAs. Tablet computers and headsets were used to enhance participant comfort and ability to respond freely. ${ }^{21}$ Survey questions included household characteristics (socioeconomic status and food security), and individual demographic characteristics, sexual behaviour, screening for common mental disorders using the Shona Symptom Questionnaire and Patient Health Questionnaire (PHQ)-2, ${ }^{22}{ }^{23}$ social cohesion, history of HIV testing, uptake of HIV testing and HIV services such as VMMC, PrEP and HIV treatment. Participants in community-led arm headman units were also asked about whether they played any role in the intervention activities. In addition, we collected dried blood spot specimens that we tested for HIV and viral load. Participants could collect DBS results from the local clinic and receive post-test services according to local standard of care. Collection of results was voluntary and no follow-up was made in the event that people did not link.

During community-engagement meetings and HIVST distribution in the community-led arm, trained social science researchers conducted participant observations, capturing interactions during meetings and activities, interactions and community atmosphere during HIVST distribution.

\section{Difference-in-differences substudy}

This was a prespecified substudy. To assess impact of HIVST and the community-led intervention on ART initiation at local health facilities, we extracted data on monthly ART initiations from registers at all facilities in the six districts for the periods 6 months before HIVST distribution, during distribution and 3 months post distribution, collecting ART initiation data from facilities whose catchment areas were in either HIVST arm or that had had no HIVST distribution.

\section{Costing}

The full provider's costs of delivering both intervention arms observed over the full implementation period were analysed. The analysis combined financial costing, using top-down allocation of actual expenses in a stepwise fashion to study arms activity, and sites using established allocation factors. ${ }^{11}$ We include above service level costs and direct cost of service provision including training, self-test distribution and support and supervision. The economic costing supplemented the financial analysis with bottom-up costing to value donated goods or services, including valuing community in-kind contributions. Valuation of distributor time in the paid distributor applied the value of the stipend (US $\$ 50$ per distributor), in the community-led model we costed both the direct financial and economic (in-kind) contribution of the headman unit. We present total and unit cost per kit distributed. As the literature has shown that programme costs change over time, we recognise that the paid distributor arm was an established model, while the community-led model is new. To put this in context we compare our costs with the costs of the paid distributor model in 2016/2017 when it was new. All costs are presented in 2020 US\$.

\section{Outcomes}

The trial had three primary outcomes, the first two measured at the individual level based on self-report among survey respondents:

1. Proportion satisfying the composite outcome of selfreported linkage to confirmatory testing (among those with a reactive (positive) self-test), VMMC or PrEP, among self-testers; 
2. Proportion newly diagnosed with HIV, among all respondents;

3. Cost per person tested with HIVST.

\section{Statistical methods}

Sample size calculation

We assumed 300 survey participants per headman unit, with $90 \%$ not on ART at study start. Twenty headman units per arm would then provide $90 \%$ power to detect a $40 \%$ increase in linkage from $10 \%$ in the paid distribution arm to $14 \%$ in the community-led arm. Assuming $1 \%$ of paid distribution arm survey participants reported newly diagnosed HIV, then 20 headman units per arm would provide $90 \%$ power to detect an increase to $2 \%$ in the community-led arm. Both sample size calculations assume a cluster coefficient of variation $(\mathrm{k})$ of 0.25 .

\section{Data analysis}

An analysis plan was finalised before data collection was complete. All analysis used Stata V.14. Before primary outcome analysis, we compared similarity by arm for prespecified variables to identify substantial differences that would need adjustment. Both primary outcomes of proportion (newly diagnosed HIV and linkage to posttest services) were analysed using mixed effect logistic regression with random effects adjustment for clustering at headman unit level. Based on identified differences, we adjusted for household food insecurity, religion and education, which were included as fixed effects in the adjusted model. A prespecified subgroup analysis investigated whether the effect of the community-led intervention on newly diagnosed HIV and linkage outcomes differed by age, sex, religion, education and household food insecurity. Household food insecurity was assessed according to the Household Food Insecurity Access Scale. ${ }^{24}$

The first primary outcome, proportion newly diagnosed with HIV, was defined as first positive test plus a provider-confirmed positive test since the start date of HIVST distribution among all surveyed participants. For the second outcome the denominator was number of surveyed participants who reported testing themselves for HIV using a HIVST kit and not taking antiretroviral drugs (ARVs) at the time of testing themselves for HIV. The numerator was based on participant confirmation of taking up any of the three post-test services of confirmatory testing, VMMC or PrEP, following self-testing.

For the difference-in-differences study, generalised estimation equations were used to analyse the relationship between ART initiation rates with (1) the periods before, during and after HIVST distribution, (2) study arm and (3) whether HIVST was distributed in the clinic catchment area for either study arm. We adjusted for district and calendar period. In a post-hoc analysis we compared ART initiation rates by distribution period across all health facilities regardless of whether HIVST were distributed locally.
Study progress was reviewed every 6 months by an independent Technical Advisory Group.

\section{Patient and public involvement}

Before the trial we held meetings with headman units to get views on how community-led HIVST models could be planned and implemented. During trial implementation we worked closely with the leadership in each headman unit, getting their permission before activities began. At the end of the trial we conducted a learning event where we invited headman units to report on how they had implemented community-led HIVST and how it could be scaled in the future.

\section{RESULTS}

The trial (inclusive of HIVST distribution and the survey) was conducted between October 2018 and December 2019. All headman units in each arm received the intended treatment (figure 1) and data from all headman units was used in the analysis of outcomes. In the community-led arm 27812 HIVST kits were distributed, with a range of 28-159 kits distributed per distributor, compared with 36699 kits and a range of 50-375 kits per distributor, respectively, in the paid distributor arm. In the community-led arm, 5 headman units opted to only distribute kits door-to-door, while in the other 15 headman units kits could be collected from various locations including distributor homes, meeting venues or other locations in the headman unit. No social harms were reported in either arm, although $11.6 \%$ of those who received kits reported in the survey that they did not feel able to turn down the offer of the HIVST, and this did not differ by arm.

The post-intervention surveys included 11150 participants, of whom 5683 were in the community-led arm. The overall survey response rate was $84.5 \%$ and did not differ by arm. Table 1 shows the characteristics of surveyed participants, which were mostly similar by arm.

HIVST uptake was lower in the community-led arm: of surveyed participants 1205 (21.6\%) took up HIVST in the community-led arm, compared with $1532(27.5 \%)$ in the paid distributor arm, adjusted OR (aOR) $0.71(95 \%$ CI 0.50 to 1.01 ), $\mathrm{p}=0.06$.

\section{Effect of community-led HIVST on primary outcomes}

We found no difference in linkage to post-test services: in the community-led arm, 318 of 1229 (25.9\%) of participants who used self-test kits linked to confirmatory HIV testing, VMMC or PrEP compared with 361 of 1508 $(23.9 \%)$ in the paid distribution arm, aOR 1.1 (0.75 to 1.49) (table 2). The calculated $\mathrm{k}$ was 0.31 . In the community-led arm, 211 of 5683 (3.7\%) of surveyed participants reported having received an HIV diagnosis after the first date of HIVST distribution in their headman unit, compared with 197 of $5467(3.6 \%)$ in paid distributor arm, aOR 1.1 ( 0.72 to 1.56$)$ ), with $\mathrm{k}=0.51$.

In the prespecified subgroup analysis, there was a trend towards better linkage to post-test services among men in 
Table 1 Characteristics of respondents in population-based survey

\begin{tabular}{|c|c|c|c|c|c|c|}
\hline & \multicolumn{2}{|c|}{ Paid distributor arm } & \multicolumn{2}{|c|}{ Community-led arm } & \multicolumn{2}{|l|}{ Total } \\
\hline & $\mathbf{n} / \mathbf{n}$ & $\%$ & $\mathrm{n} / \mathrm{n}$. & $\%$ & $\mathbf{n} / \mathbf{n}$. & $\%$ \\
\hline Clusters (No.) & 20 & 50 & 20 & 50 & 40 & 100 \\
\hline Adult household members (No.) & 6442 & 48.8 & 6748 & 51.2 & 13190 & 100 \\
\hline $\begin{array}{l}\text { Mean number of adult household } \\
\text { members per headman unit }\end{array}$ & 322.1 & & 337.4 & & 329.8 & \\
\hline $\begin{array}{l}\text { Mean number of surveyed } \\
\text { respondents per headman unit }\end{array}$ & 273.4 & & 284.2 & & 278.8 & \\
\hline \multicolumn{7}{|l|}{ Household characteristics } \\
\hline $\begin{array}{l}\text { Adult members per household (mean/ } \\
\text { SD) }\end{array}$ & \multicolumn{2}{|l|}{$2.1(1.1)$} & \multicolumn{2}{|l|}{$2.2(1)$} & \multicolumn{2}{|l|}{$2.1(1.1)$} \\
\hline Poorest 1 & $536 / 2736$ & 19.6 & $579 / 2836$ & 20.4 & $1115 / 5572$ & 20 \\
\hline Second 2 & $596 / 2736$ & 21.8 & $530 / 2836$ & 18.7 & $1126 / 5572$ & 20.2 \\
\hline Middle 3 & $556 / 2736$ & 20.3 & $547 / 2836$ & 19.3 & $1103 / 5572$ & 19.8 \\
\hline Fourth 4 & $534 / 2736$ & 19.5 & $580 / 2836$ & 20.5 & $1114 / 5572$ & 20 \\
\hline Least poor 5 & $514 / 2736$ & 18.8 & $600 / 2836$ & 21.2 & $1114 / 5572$ & 20 \\
\hline \multicolumn{7}{|l|}{ Household food insecurity $(\mathrm{n} / \mathrm{n}(\%)) \S$} \\
\hline Severe household hunger & $572 / 2776$ & 20.6 & $525 / 2850$ & 18.4 & $1097 / 5626$ & 19.5 \\
\hline Moderate household hunger & $951 / 2776$ & 34.3 & $945 / 2850$ & 33.2 & $1896 / 5626$ & 33.7 \\
\hline Little-to-no household hunger & $1253 / 2776$ & 45.1 & $1380 / 2850$ & 48.4 & $2633 / 5626$ & 46.8 \\
\hline \multicolumn{7}{|l|}{ Marital status (n/n (\%)) ** } \\
\hline Married or living as married & $3397 / 5394$ & 63 & $3364 / 5601$ & 60.1 & $6761 / 10995$ & 61.5 \\
\hline Never married & $1235 / 5394$ & 22.9 & $1366 / 5601$ & 24.4 & 2601/10 995 & 23.7 \\
\hline Widowed/separated/divorced & $762 / 5394$ & 14.1 & $871 / 5601$ & 15.6 & $1633 / 10995$ & 14.9 \\
\hline \multicolumn{7}{|l|}{ Highest level of education (n/n (\%)) } \\
\hline Primary complete or less & $2338 / 5467$ & 42.8 & $2038 / 5683$ & 35.9 & $4376 / 11150$ & 39.2 \\
\hline Some secondary education & $1367 / 5467$ & 25 & $1441 / 5683$ & 25.4 & 2808/11 150 & 25.2 \\
\hline $\begin{array}{l}\text { Secondary education complete or } \\
\text { higher }\end{array}$ & $1762 / 5467$ & 32.2 & $2204 / 5683$ & 38.8 & $3966 / 11150$ & 35.6 \\
\hline \multicolumn{7}{|l|}{ Religion (n/n (\%)) } \\
\hline Apostolic & $2206 / 5467$ & 40.4 & $2055 / 5683$ & 36.2 & $4261 / 11150$ & 38.2 \\
\hline Other & $3261 / 5467$ & 59.6 & $3628 / 5683$ & 63.8 & $6889 / 11150$ & 61.8 \\
\hline Receives regular salary $(\mathrm{n} / \mathrm{n}(\%)) \dagger \dagger$ & $1313 / 5401$ & 24.3 & $1263 / 5618$ & 22.5 & $2576 / 11019$ & 23.4 \\
\hline \multicolumn{7}{|c|}{$\begin{array}{l}\text { *120 households missing household member information in paid distributor arm and } 127 \text { in community-led arm. } \\
\text { †176 households missing assets data in paid distributor arm and } 164 \text { in community-led arm. } \\
\text { †Ascertained at the household level using an assets index generated using principal components analysis. } \\
\S 136 \text { missing hunger information in paid distributor arm and } 150 \text { in community-led arm. } \\
\text { १3 missing age in paid distributor arm and } 5 \text { in community-led arm. } \\
\star * 73 \text { missing marital status in paid distributor arm and } 82 \text { in community-led arm. } \\
\dagger † 66 \text { missing salary data in paid distributor arm and } 65 \text { in community-led arm. }\end{array}$} \\
\hline
\end{tabular}


Table 2 Primary outcomes

\begin{tabular}{|c|c|c|c|c|c|c|}
\hline & \multicolumn{2}{|c|}{ Paid distributor arm } & \multicolumn{2}{|c|}{ Community-led arm } & \multirow[b]{2}{*}{ OR (95\% Cl) } & \multirow[b]{2}{*}{ P value } \\
\hline & $\mathrm{n} / \mathrm{n}$ & $\%$ & $\mathrm{n} / \mathrm{n}$ & $\%$ & & \\
\hline \multicolumn{7}{|c|}{ Primary outcome 1: self-reported linkage to confirmatory testing, PrEP and VMMC } \\
\hline Unadjusted & $361 / 1508$ & $23.9 \%$ & $318 / 1229$ & $25.9 \%$ & $1.1(0.80$ to 1.48$)$ & 0.6 \\
\hline Adjusted & & & & & $1.1(0.75$ to 1.49$)$ & 0.8 \\
\hline \multicolumn{7}{|c|}{ Primary outcome 2: proportion of individuals reporting a new HIV diagnosis } \\
\hline Unadjusted & $197 / 5467$ & $3.6 \%$ & $211 / 5683$ & $3.7 \%$ & 1 (0.69 to 1.52$)$ & 0.9 \\
\hline Adjusted & & & & & $1.1(0.72$ to 1.56$)$ & 0.8 \\
\hline
\end{tabular}

the community-led arm with ORs of 1.28 (0.87 to 1.88 ) and 0.84 (0.57 to 1.24$)$ among men and women, respectively, interaction $\mathrm{p}=0.02$ (table 3 ). No other differences were seen in the subgroup analysis.

\section{Results from difference-in-differences study}

We collected data on monthly ART initiations from 133 clinics, including 46 with HIVST in their catchment areas, with a total of 1330 clinic-months of follow-up spanning the pre-intervention period (798 clinic months), intervention period ( 130 clinic months) and post-intervention period (403 clinic months), and a total of 5302 ART initiations (table 4). Although the HIVST distribution period was associated with higher ART initiation rates, differences-in-differences analyses show no significant differences when comparing HIVST versus non-HIVST facilities, and a reduction in initiation rate ratio (IRR) when comparing community-led versus paid distributor arms, with interaction IRR of $1.13(0.98-1.32), \mathrm{p}=0.1$ and $0.76(0.60-0.96), \mathrm{p}=0.02$, respectively, during the intervention period (table 4). In post-hoc analysis, ART initiations increased significantly during HIVST distribution across all facilities, including those not in HIVST areas, aOR 1.30 (1.24 to 1.37), falling to baseline levels postdistribution.

\section{Costing}

Total distribution costs were calculated as US\$231 212 and US\$285 065 for paid distributor and community-led programmes, respectively (online supplemental appendix 1). Human resources were the largest cost category in both model arms at $39 \%$ and $46 \%$, followed by HIVST kits which contributed $37 \%$ and $23 \%$, respectively. Vehicle costs were $6 \%$ and $8 \%$ of total costs, respectively. The cost per HIVST kit distributed was US\$6.29 and US\$10.25 for the paid distributor arms and community-led arms, respectively. When the paid distributor model was first introduced in 2016/17, the cost per HIVST kit distributed was $\$ 14.52$. $^{11}$

Across sites the cost per HIVST kit distributed ranged from US\$5.49 to U\$9.52 in the paid distributor arm and US\$6.14 to US\$33.11 in the community-led model arm. Unit costs were generally lower at sites with larger numbers of self-test kits distributed, suggesting a spreading of fixed costs across variable numbers of kits (ie, economies of scale).

\section{DISCUSSION}

In this cluster-randomised trial comparing community-led distribution of HIVST kits against communitybased distribution by paid distributors in 40 rural Zimbabwean communities (headman units), we found no difference in self-reported number of new HIV diagnosis nor self-reported linkage to post-test services (confirmatory testing, VMMC or PrEP). There was also no difference in ART initiation rates at health facilities in the study districts by HIVST distribution model, although a post-hoc analysis showed a 30\% increase in ART initiation rates during the HIVST distribution period across all health facilities in line with previous research from Zimbabwe and Malawi showing that community provision of HIVST can increase rate of ART initiations. Our subgroup analyses suggested that men may be more likely to link to post-test services in the community-led model. Of note, the cost per HIVST kit distributed in the community-led arm was US $\$ 10.25$, which was higher than paid distribution (US\$6.30). The higher cost of community-led distribution may in part reflect inexperience with this distribution model, as costs in both arms were lower than for paid distribution of HIVST kits when first implemented in 2016/2017 (US\$14.52). ${ }^{11}$ Thus, as experience with the paid distributor model increased, implementation costs reduced by $57 \%$.

There is evidence of the acceptability, safety and feasibility of community-led HIVST. ${ }^{25} 26$ The trial adds evidence that communities are able to organise themselves to design and implement context-specific HIVST distribution models that achieve similar rates of coverage and uptake to those achieved by professionally supervised paid distributors. Our process evaluation data (not presented here) suggests that most communities were glad to be involved and were highly motivated to distribute HIVST kits. It is encouraging that the cost per kit distributed in the community-led model was lower than that in the paid distributor model when first introduced. Implementation of the professionally supervised paid distributor model has become more efficient over 


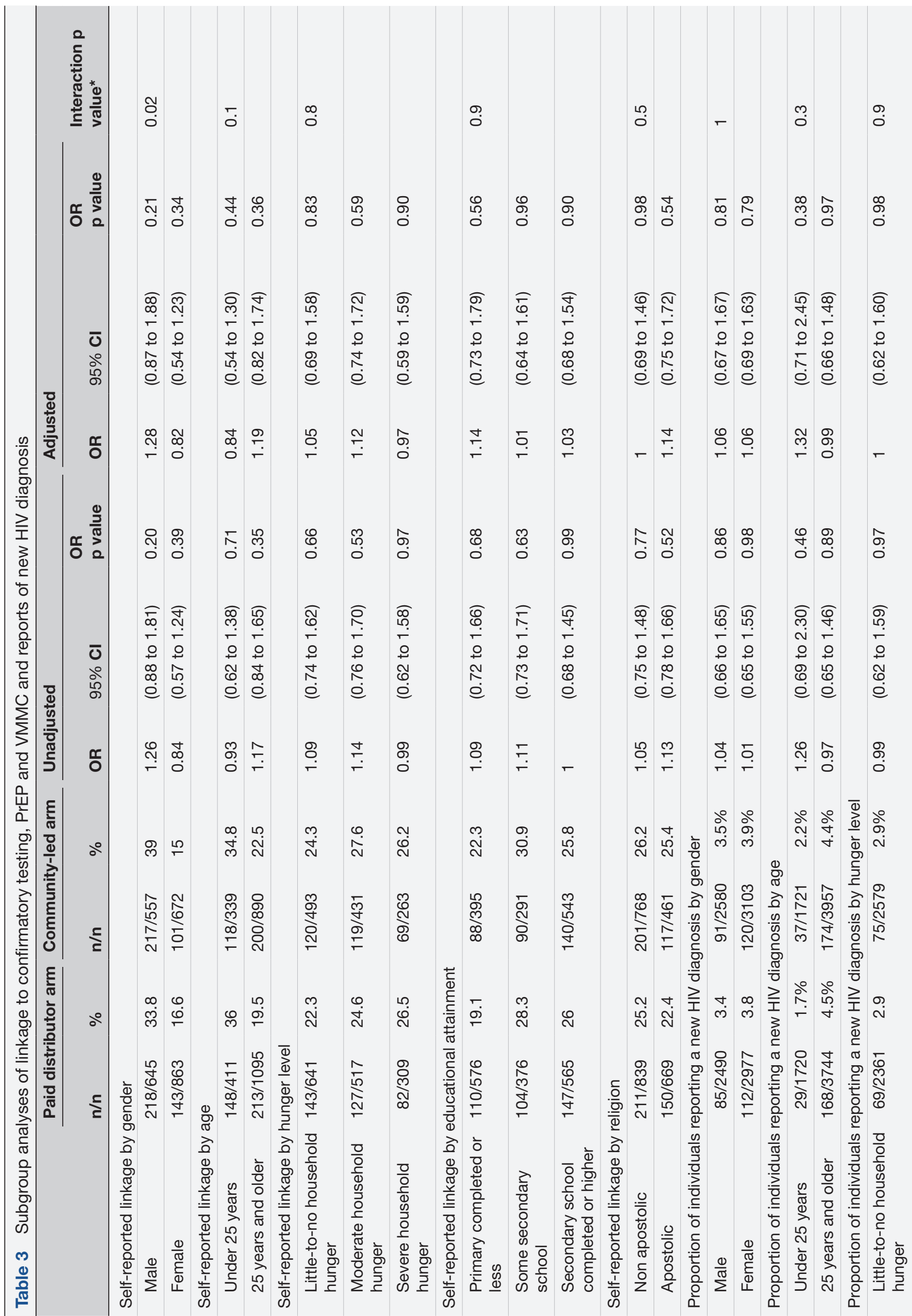




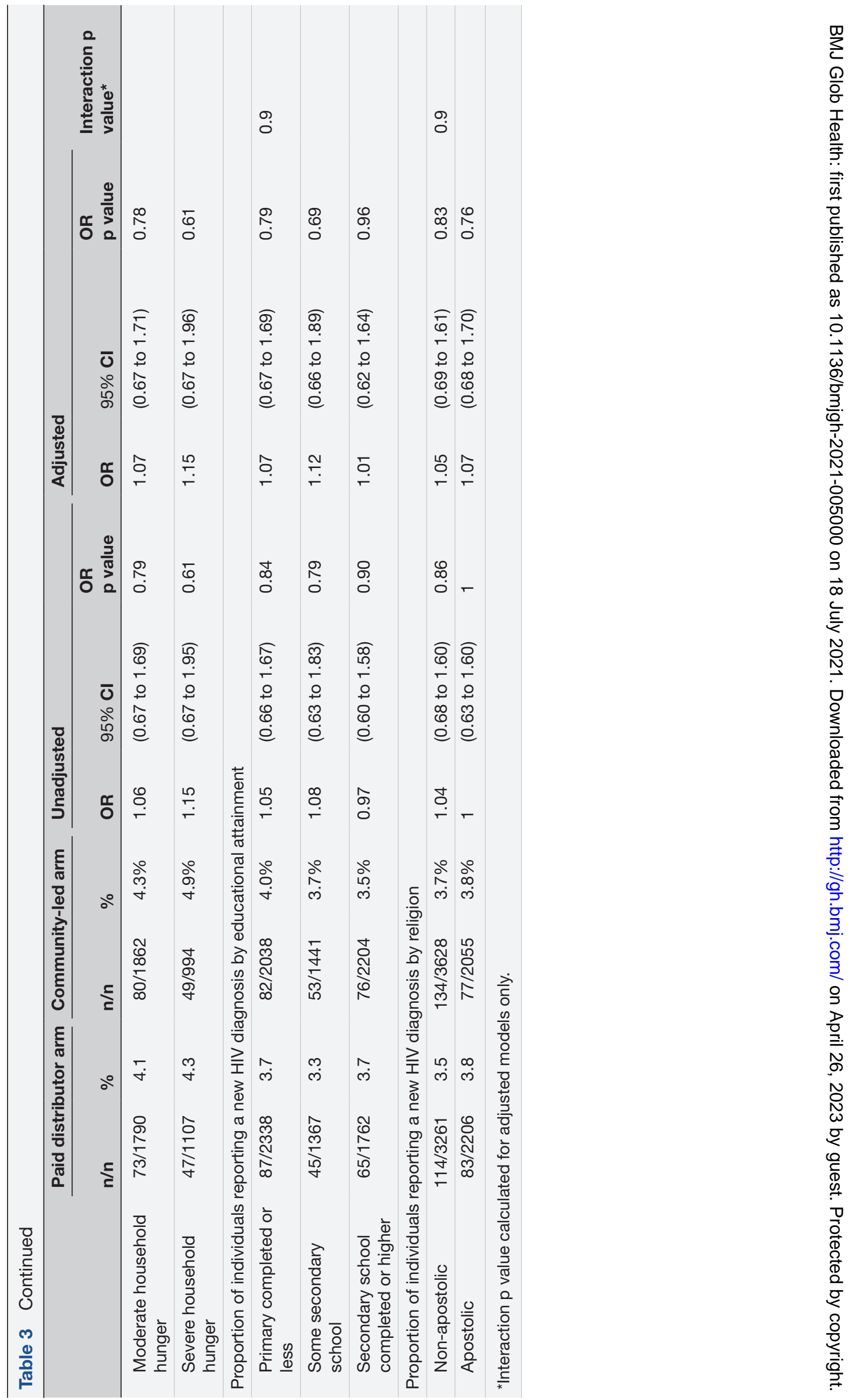




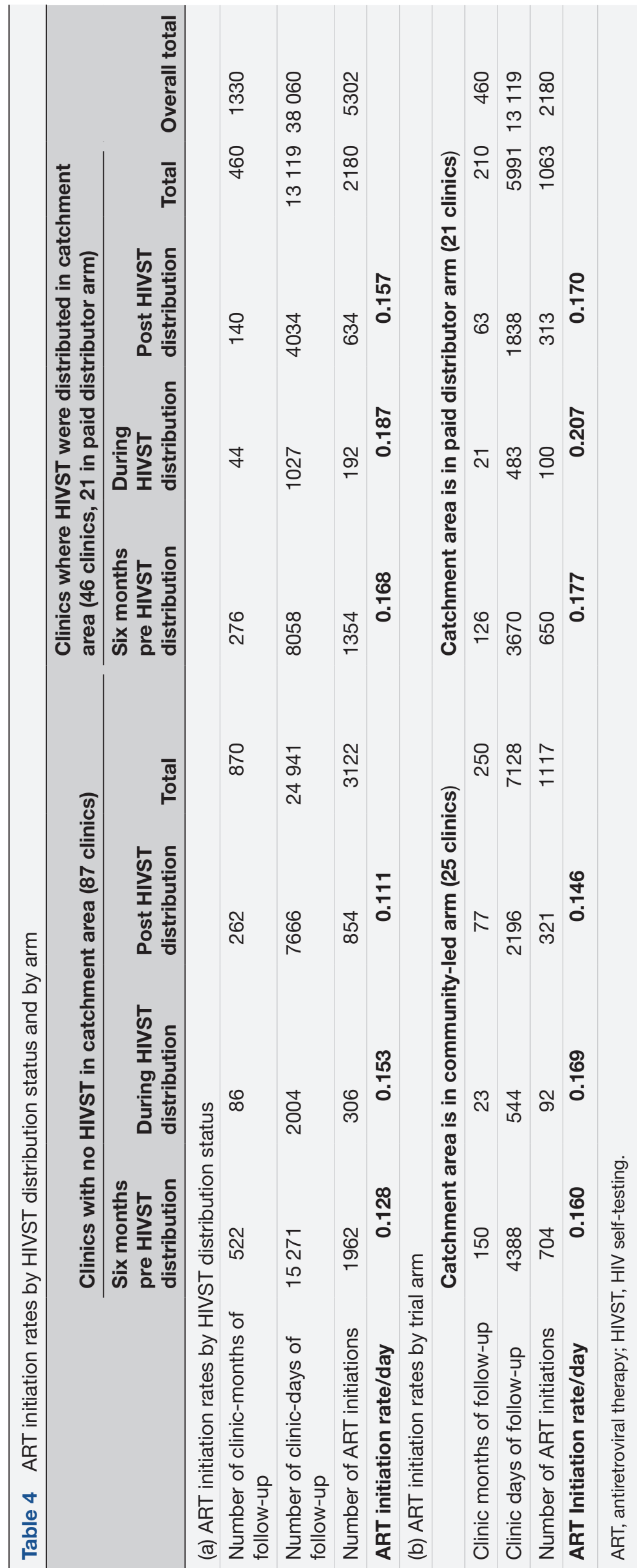

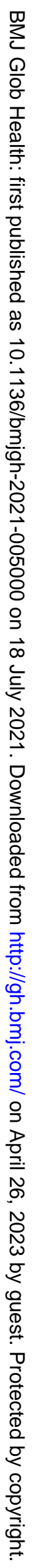


time leading to reduced costs and it is likely that the same would be true of the community-led approach. Given the acceptability and feasibility of engaging communities to distribute self-test kits, and the success of other community-led interventions ${ }^{13} 1427$ in engaging communities to take ownership of their own healthcare, future research could evaluate using a community-led approach to layer a range of health and welfare activities (self-care) alongside HIVST.

As countries get closer to achieving their UNAIDS Fast Track 95-95-95 targets, identifying people who are unaware of their status will become more difficult and more costly. Novel approaches and testing strategies will be required to efficiently reach those who have traditionally been hard to reach with testing services. Importantly, distribution of HIVST needs to be accompanied by ongoing efforts to promote linkage to post-test services. In this study distributors were trained to provide information and encouragement for linkage when distributing kits. Other research has found that initiatives to promote linkage, such as provision of community-based services, can improve linkage following testing. ${ }^{28}$ The community-led model we evaluated, which is integrated with the local health system, can potentially be scaled up long term. Costs, which are encouragingly lower than newly introduced paid distributor models, are likely to come down not only because of programme learning, but with use of public sector human resources, such as community health workers (rather than the more expensive PSI workers) for community engagement. That the subgroup analysis showed a trend towards better linkage to posttest services in men is encouraging and suggests that this could be taken forward to optimise care in this group, whose uptake of HIV services has been known to be suboptimal. ${ }^{29}$

Contrary to findings from our previous studies in Malawi and Zimbabwe, ${ }^{10}$ we did not show a significant difference in ART initiation rates at health facilities whose catchment areas had community-based HIVST, when compared with those where no HIVST was distributed. We believe this likely reflects a much smaller unit of evaluation (headman unit of 500-4000 people) in this study than the typical health facility catchment populations. Thus, catchment areas where HIVST was distributed were only partially covered. The findings of the post-hoc analysis, where ART initiation rates increased during the HIVST distribution period across health facilities, are credible given the higher than expected numbers of new HIV diagnoses in both arms of the study (twice the $2 \%$ we expected during study design), which is in line with our previous findings of the effectiveness of HIVST in increasing ART initiation. This emphasises the potential utility of HIVST to increase status awareness.

The strengths of this study include that we evaluated an acceptable, feasible and potentially scalable community model of HIVST kit distribution that was rooted within the public sector system. We used robust methods in the design, implementation and analysis of the trial and difference-in-differences analysis. Limitations include the use of self-reports for primary outcomes, although we tried to minimise the self-report bias by use of ACASI. Our unit of implementation, the headman unit, was much smaller than the average health facility catchment area, which limited our ability to detect an effect of HIVST on ART initiation rates. However the trend towards increase in ART initiation rates as shown by the post-hoc analysis compare well with our previous findings. ${ }^{10}$ Costing from the provider's perspective does not take account of the resources that headman units contributed to the successful implementation of the intervention and this may undermine sustainability in practice.

In summary, in this cluster randomised trial we found that communities can organise themselves to design and implement community-based HIVST distribution with outcomes that are comparable to programmes where distributors are professionally supervised and paid. The community-led HIVST intervention is a promising model that can be integrated with local standard of care and for scale up. The cost per HIVST kit distributed was lower than that for the paid distributor model when it was first introduced; programme costs are likely to reduce as programmes become embedded in communities and become more efficient, making the model sustainable.

\section{Author affiliations}

${ }^{1}$ CeSHHAR Zimbabwe, Harare, Zimbabwe

${ }^{2}$ Department of International Public Health, Liverpool School of Tropical Medicine, Liverpool, UK

${ }^{3}$ Department of Infectious Disease Epidemiology and MRC International Statistics and Epidemiology Group, London School of Hygiene and Tropical Medicine, London, UK

${ }^{4}$ Department of Sexual Reproductive Health Rights and Innovations, Population Services International Zimbabwe, Harare, Zimbabwe

${ }^{5}$ Faculty of Health Sciences, University of the Witwatersrand, Johannesburg, South Africa

${ }^{6}$ HIV, Hepatitis and STI Department, World Health Organisation, Geneva, Switzerland ${ }^{7}$ AIDS and TB Unit, Ministry of Health and Child Care, Harare, Zimbabwe

${ }^{8}$ Population Services International, Washington, District of Columbia, USA

${ }^{9}$ Department of Clinical Research, London School of Hygiene \& Tropical Medicine, London, UK

${ }^{10}$ TB-HIV Group, Malawi-Liverpool-Wellcome Trust Clinical Research Programme, Blantyre, Malawi

${ }^{11}$ Department of Global Health \& Development, Faculty of Public Health and Policy, London School of Hygiene \& Tropical Medicine, London, UK

${ }^{12}$ Joint United Nations Programme on HIV/AIDS, Geneva, Switzerland

Twitter Euphemia Lindelwe Sibanda @Euphemia4, Collin Mangenah @cmangenah01, Mary Tumushime @MTumushime, Cheryl Johnson @ccasejohn and Miriam Taegtmeyer @MiriamTaegtmeye

Acknowledgements We thank all participants and staff, and the STAR technical advisory group (Peter Godfrey-Faussett, Ruanne Barnabas, Vincent Wong, Mohammed Lamorde).

Contributors Formulated the research study and design: FMC, ELC, MTa, KH, CJ, ELS, GN, KLF, MN and FT-P. Informed development of data collection methods and collected data: FMC, ELC, MTu, MTa, MN, JD, MM, ELS, GN, CW, CM and GM. Analysed the data or contributed to the analysis: MN, ELS, KLF, CM, FT-P and GM Substantially provided intellectual input to the manuscript: FMC, ELC, KH, FT-P, KLF, MTu, MTa, CJ, MN, MM, CM and CW.

Funding This study was funded by Unitaid (STAR Initiative), subagreement number 4214-CeSHHAR. ELC is funded by Wellcome Trust (WT200901/Z/16/Z).

Competing interests None declared. 
Patient consent for publication Not required.

Ethics approval The study was approved by the Medical Research Council of Zimbabwe, reference number MRCZ/A/2323; London School of Hygiene \& Tropical Medicine Ethics Committee, reference number 15801; and WHO Ethical Review Committee, reference number ERC.0003065. Verbal consent for participation in the community-led arm was given by headman unit leaders. Survey participants gave written informed consent.

Provenance and peer review Not commissioned; externally peer reviewed.

Data availability statement Data are available upon request. De-identified data are available. For access please contact the principal investigator, euphemia@ ceshhar.co.zw. Protocol and statistical analysis plan are available.

Supplemental material This content has been supplied by the author(s). It has not been vetted by BMJ Publishing Group Limited (BMJ) and may not have been peer-reviewed. Any opinions or recommendations discussed are solely those of the author(s) and are not endorsed by BMJ. BMJ disclaims all liability and responsibility arising from any reliance placed on the content. Where the content includes any translated material, BMJ does not warrant the accuracy and reliability of the translations (including but not limited to local regulations, clinical guidelines, terminology, drug names and drug dosages), and is not responsible for any error and/or omissions arising from translation and adaptation or otherwise

Open access This is an open access article distributed under the terms of the Creative Commons Attribution IGO License (CC BY NC 3.0 IGO), which permits use, distribution,and reproduction in any medium, provided the original work is properly cited. In any reproduction of this article there should not be any suggestion that WHO or this article endorse any specific organization or products. The use of the WHO logo is not permitted. This notice should be preserved along with the article's original URL.

Disclaimer: The author is a staff member of the World Health Organization. The author alone is responsible for the views expressed in this publication and they do not necessarily represent the views, decisions or policies of the World Health Organization

\section{ORCID iDs}

Euphemia Lindelwe Sibanda http://orcid.org/0000-0003-1754-1076

Collin Mangenah http://orcid.org/0000-0002-0733-0622

Melissa Neuman http://orcid.org/0000-0002-8870-6504

Katherine L Fielding http://orcid.org/0000-0002-6524-3754

Cheryl Johnson http://orcid.org/0000-0001-5499-5523

Miriam Taegtmeyer http://orcid.org/0000-0002-5377-2536

Elizabeth Lucy Corbett http://orcid.org/0000-0002-3552-3181

Fern Terris-Prestholt http://orcid.org/0000-0003-1693-5196

Frances M Cowan http://orcid.org/0000-0003-3087-4422

\section{REFERENCES}

1 World Health Organisation. Consolidated guidelines on HIV testing services for a changing epidemic. 2019. Geneva, Switzerland: WHO, 2019.

2 World health Organisation. Guidelines on HIV self-testing and partner notification: supplement to consolidated guidelines on HIV testing services. Geneva, Switzerland, 2016.

3 Choko AT, Corbett EL, Stallard N, et al. Hiv self-testing alone or with additional interventions, including financial incentives, and linkage to care or prevention among male partners of antenatal care clinic attendees in Malawi: an adaptive multi-arm, multi-stage cluster randomised trial. PLoS Med 2019;16:e1002719.

4 Amstutz A, Lejone TI, Khesa L, et al. Home-Based oral self-testing for absent and declining individuals during a door-to-door HIV testing campaign in rural Lesotho (HOSENG): a cluster-randomised trial. Lancet HIV 2020;7:e752-61.

5 Mulubwa C, Hensen B, Phiri MM, et al. Community based distribution of oral HIV self-testing kits in Zambia: a clusterrandomised trial nested in four HPTN 071 (PopART) intervention communities. Lancet HIV 2019;6:e81-92.

6 Chamie G, Kamya MR, Petersen ML, et al. Reaching 90-90-90 in rural communities in East Africa: lessons from the sustainable East Africa research in community health trial. Curr Opin HIV AIDS 2019;14:449-54.

7 Floyd S, Shanaube K, Yang B, et al. Hiv testing and treatment coverage achieved after 4 years across 14 urban and peri-urban communities in Zambia and South Africa: an analysis of findings from the HPTN 071 (PopART) trial. PLoS Med 2020;17:e1003067.

8 Indravudh PP, Sibanda EL, d'Elbée M, et al. 'I will choose when to test, where I want to test': investigating young people's preferences for HIV self-testing in Malawi and Zimbabwe. AIDS 2017;31 Suppl 3:S203-12.

9 Sibanda EL, d'Elbée M, Maringwa G, et al. Applying user preferences to optimize the contribution of HIV self-testing to reaching the "first 90" target of UNAIDS Fast-track strategy: results from discrete choice experiments in Zimbabwe. J Int AIDS Soc 2019;22 Suppl 1:e25245.

10 et alSibanda EL, Neuman M MT, Hatzold K. Linkage to care after HIV self-testing in Zimbabwe: a cluster-randomised trial. Conference on Retroviruses and opportunistic infections (CROI 2018); 07 Mar 2018, 2018.

11 Mangenah C, Mwenge L, Sande L, et al. Economic cost analysis of door-to-door community-based distribution of HIV self-test kits in Malawi, Zambia and Zimbabwe. J Int AIDS Soc 2019;22 Suppl 1:e25255.

12 CDI Study Group. Community-directed interventions for priority health problems in Africa: results of a multicountry study. Bull World Health Organ 2010;88:509-18.

13 Roungou J-B, Yameogo L, Mwikisa C, et al. 40 years of the apoC partnership. PLoS Negl Trop Dis 2015;9:e0003562.

14 Zimba R, Ngulube V, Lukama C, et al. Chiengi district, Zambia open defecation free after 1 year of Community-Led total sanitation. Am J Trop Med Hyg 2016;95:925-7.

15 SEARCH Collaboration. Evaluating the feasibility and uptake of a community-led HIV testing and multi-disease health campaign in rural Uganda. J Int AIDS Soc 2017;20:21514.

16 STAR Project. Hiv Self-testing Africa project website. Available: http://hivstar.Ishtm.ac.uk/

17 Zimbabwe National Statistics Agency (ZIMSTAT), ICAP. Zimbabwe population-based impact assessment (ZIMPHIA 2020). Harare, Zimbabwe: ICAP, 2020.

18 Hoffmann TC, Glasziou PP, Boutron I, et al. Better reporting of interventions: template for intervention description and replication (TIDieR) checklist and guide. BMJ 2014;348:g1687.

19 Cohen MS, Chen YQ, McCauley M, et al. Prevention of HIV1 infection with early antiretroviral therapy. $N$ Engl J Med 2011;365:493-505.

20 Napierala-Mavedzenge S, Sibanda EL, Mavengere Y. Supervised HIV self-testing to inform implementation and scale up of self-testing in Zimbabwe. In: The 8th IAS conference on HIV pathogenesis, treatment and prevention (IAS 2015) July 2015. Vancouver, Canada. Vancouver, Canada: IAS, 2015.

21 Langhaug LF, Cheung YB, Pascoe SJS, et al. How you ask really matters: randomised comparison of four sexual behaviour questionnaire delivery modes in Zimbabwean youth. Sex Transm Infect 2011;87:165-73.

22 Patel V, Simunyu E, Gwanzura F, et al. The Shona symptom questionnaire: the development of an Indigenous measure of common mental disorders in Harare. Acta Psychiatr Scand 1997;95:469-75.

23 Kroenke K, Spitzer RL, Williams JBW. The patient health Questionnaire-2: validity of a two-item depression screener. Med Care 2003;41:1284-92.

24 Deitchler M, Ballard T, Swindale A. Validation of a measure of household hunger for CrossCultural use. Washington, DC: FHI, 2010: 360.

25 Indravudh PP, Fielding K, Kumwenda MK. Community-led delivery of HIV self-testing targeting adolescents and men in rural Malawi: a cluster-randomised trial. Mexico City, Mexico: IAS, 2019.

26 Nguyen VTT, Phan HT, Kato M, et al. Community-led HIV testing services including HIV self-testing and assisted partner notification services in Vietnam: lessons from a pilot study in a concentrated epidemic setting. J Int AIDS Soc 2019;22 Suppl 3:e25301.

27 Andersson N, Nava-Aguilera E, Arosteguí J, et al. Evidence based community mobilization for dengue prevention in Nicaragua and Mexico (Camino Verde, the green way): cluster randomized controlled trial. BMJ 2015;351:h3267.

28 MacPherson P, Lalloo DG, Webb EL, et al. Effect of optional home initiation of HIV care following HIV self-testing on antiretroviral therapy initiation among adults in Malawi: a randomized clinical trial. JAMA 2014;312:372-9.

29 Joint United Nations Programme on HIV/AIDS (UNAIDS). 2020 global AIDS update. Geneva, Switzerland: UNAIDS, 2020. 